\title{
Loss of genetic material within $1 p$ and $19 q$ chromosomal arms in low grade gliomas of central nervous system
}

\author{
Daniel Ręcławowicz', Mirosław Stempniewicz', Wojciech Biernat², Janusz Limon ${ }^{3}$, Paweł Słoniewski ${ }^{1}$ \\ ${ }^{1}$ Neurosurgery Department, Medical University of Gdansk, 2Pathomorphology Department, Medical University of Gdansk, ${ }^{3}$ Department \\ of Biology and Genetics, Medical University of Gdansk, Poland
}

\begin{abstract}
Diffuse gliomas can constitute up to one third of all gliomas diagnosed in neurosurgical centers. Their invasive growth, progression to more malignant lesions, and the lack of standardized management guidelines render a significant clinical problem. The discovery of $1 p$ and $19 q$ chromosomal arms deletion in neoplastic cells will probably influence both more objective diagnosis and more accurate prediction of chemotherapy response. Defining the above mentioned deletion is becoming a standard procedure in Western European countries and in the USA when LGG is diagnosed. As a result an attempt has been made to detect deletion using fluorescence in situ hybridization and to determine its prognostic value.

Genetic material from 34 grade II gliomas was examined. Separate 1p and 19q deletions were discovered in 14 and 16 cases respectively. Simultaneous occurrence of both was observed in 12. The frequency of occurrence of simultaneous deletions $1 p$ and $19 q$ varied based on histopathological diagnosis. This disorder was not observed in astrocytomas, in oligoastrocytomas it appeared in 50\% cases. The highest incidence of deletion was noted in oligodendrogliomas and amounted to $66.7 \%, p<0.005$. Median survival in patients with diagnosed $1 p$ and $19 q$ deletion in their neoplastic cells is twice longer in comparison with patients in whom no such deletion was observed (80 months vs. 41 months, $p<0.05$ ). Frontal location of a tumor occurred to be a statistically significant factor unfavorable for prognosis, $p<0.05$. In the work presented the fluorescence in situ hybridization was successfully applied to identify deletion 1p/19q. Its incidence depends on the type of diagnosed glioma. Deletions also have prognostic significance in the test group what constitutes the basis for inclusion of determining deletion 1p/19q into diagnostic and treatment algorithm in LGGs.
\end{abstract}

Key words: astrocytoma, oligodendroglioma, oligoastrocytoma, low grade gliomas, 1p, $19 q$.

\section{Introduction}

The definition of low grade glioma encompasses three types of WHO grade II tumours including: diffuse astrocytoma (DA), oligodendroglioma (OD), and mixed glioma - oligoastrocytoma (OA). They constitute up to
$30 \%$ of all glial tumours of the central nervous system. Distinguishing between these three types may be challenging due to both subjectivity of pathologists' judgment and lack of reliable objective markers confirming the diagnosis. The WHO classification does not provide the exact cut-off values of the oligodendroglial 
and astrocytic component between three aforementioned types of tumours. Thus, discrepancy among pathologists exists, some use a 50\% threshold level for the oligodendroglial component in making the diagnosis of oligodendroglioma [31], whereas others use a $10 \%$ cut-off [15].

Recent publications suggest that deletion of chromosomal arm 1p/19q may play such an indicative role in the diagnosis of low grade gliomas (LGG) $[1,11]$. LGGs demonstrate a broad range of genetic disturbances. One of the frequent alterations is simultaneous deletion of the short arm of chromosome 1 and the long arm of chromosome 19 [9]. Although chromosome 19q deletion used to be regarded a feature of both oligodendrogliomas and astrocytomas, further studies have shown its predominance in the former group [28]. Both deletions of $1 p / 19 q$ frequently occur together and are seldom found as a separate alteration $[4,19,24]$. It is estimated that frequency of concurrent $1 p$ and $19 q$ deletion approaches $70-80 \%$ in oligodendrogliomas, 30-50\% in mixed gliomas and only $7-15 \%$ in astrocytomas [4,19, 24]. The deletion of $1 p / 19 q$ is referred to as the most common genetic abnormality observed in oligodendrogliomas. Therefore, $1 p / 19 q$ deletion seems to be a reliable signature of this neoplasm.

In addition, the deletion of $1 p / 19 q$ is additionally a predictive factor in OD. Cairncross et al. demonstrated that $1 p$ deletion is a marker of the tumour chemosensitivity to procarbazine, lomustine and vincristine scheme (PCV) and concurrent $1 p / 19 q$ deletion is a positive factor for a longer time to progression in anaplastic oligodendrogliomas [7]. Additionally, the use of multivariate statistics showed that survival in patients with $1 p / 19 q$ loss is statistically longer than in patients without this alteration. These results were confirmed by retrospective analyses based on clinical studies EORTC 26951 and RTOG 9402. Both proved that presence of co-deletion correlates with the extended time to progression and time do death in grade III $[3,6]$ and grade II gliomas [12]. Moreover, the latter study disclosed beneficial response to chemotherapy in patients with LOH 1p/19q.

Based on these data we performed this study at the Medical University of Gdansk in order to correlate the status of 1p/19q deletion in LGGs with tumour morphology and patients' demographic factors.

\section{Material and methods}

We retrieved tissue samples of WHO grade II gliomas from the Department of Neurosurgery and the Department of Pathology over a period from 1997 to 2002. The tissue available for study was obtained in 68 cases. Verified diagnosis was based on the 2007 WHO classification of CNS tumours (Fig. 1). LGG diagnosis was confirmed in 47 cases. Due to the small amount of tissue, neoplastic material from 39 cases was prepared for tissue microarray study (TMA) and following fluorescence in situ hybridization (FISH). During the revision process most representative parts of each tumour were marked.

Selected tissue was taken into TMA acceptor blocks using tissue arrayer (MTI, Beecher Instruments, Silver Spring, MD) with $1.5 \mathrm{~mm}$ punches. TMAs were cut into 4-micrometer slices. One slice from each array was stained H\&E for histological control.

Fluorescence in situ hybridization was performed using commercially available LSI 1p36/1q25 and LSI 19q13/19p13 Dual Color probes (Abbott Laboratories,

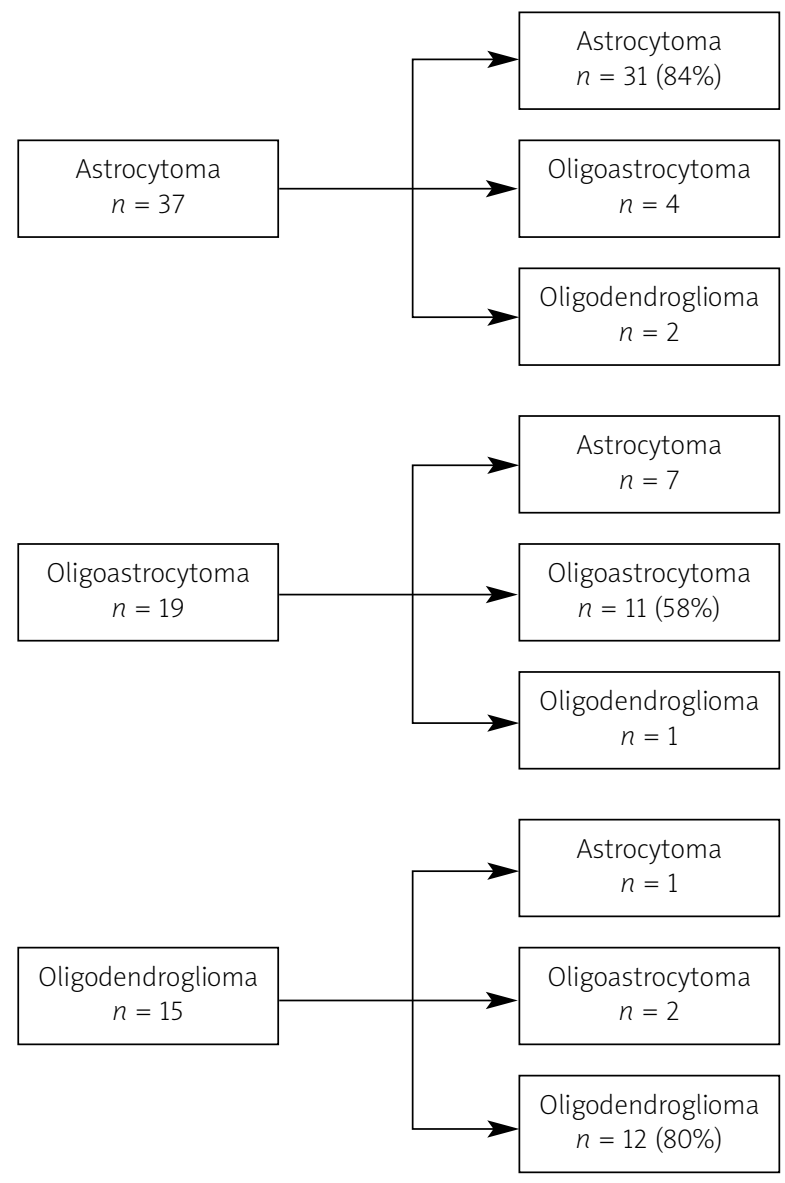

Fig. 1. Changes of primary (left) diagnoses after revision (right) and concordance percentage among the three LGG types. 
previously Vysis). The kit contains two mixtures. The first one consists of probe for locus 1p36 and control probe for 1q25. The second mixture stains an examined locus $19 q 13$ (red) and a control locus 19p13 (green). Four signals are observed in normal diploid nuclei - two red and two green ones. In 34 patients FISH proved sufficient to supply reliable results.

Signals from 50 non-overlapping nuclei were assessed and to eliminate random sectioned nuclei, deletion was diagnosed when more than $20 \%$ of examined signals in relation to control signals were absent [13]. In all borderline cases (with the absence of $15-25 \%$ of signals), additional 50 nuclei were reassessed.

The analysed group of 34 patients with LGG consisted of $21(62 \%)$ males and 13 (38\%) females. Average age at the time of operation was 40.9 years (range: 18.2-62.8; SD = 10.8). In most cases, the tumour tissue was obtained by resection. Only in 4 (11.7\%) cases, tissue from stereotactic biopsy was acquired. The histological diagnoses were as follows: diffuse astrocytoma, grade II (All; $n=12,35 \%$ ), oligoastrocytoma (OAII, $n=10 ; 30 \%$ ), oligodendroglioma (OII, $n=12 ; 35 \%)$.

Survival time was defined as time from the date of the operation to death or, in censored cases, time to exclusion of the patient death. All appropriate data were imported to Statistica (Statsoft) - statistical analysis software. Median survival times calculation and survival graphs were performed using Kaplan-Meier analysis. Impact of prognostic factors was assessed by univariate log-rank tests. In order to identify independent factors affecting survival, statistically significant factors were introduced in multivariate analysis (Cox proportional hazard model). All results were considered statistically significant when $p<0.05$.

\section{Results}

Fluorescence in situ hybridization revealed $1 p$ deletion in 14 (41\%) cases and 19q deletion in 16 cases (47\%, see Table I). Simultaneous deletion of 1p/19q was detected in 12 cases (correlation coefficient $\mathrm{Fi}^{2}=0.65$, $p<0.0002$ ).

Codeletion of $1 p / 19 q$ correlated strongly with the histological type. It was not identified in All, however, it amounted to $50 \%$ in OAll and to $66.7 \%$ in Oll ( $p=0.003$, Table I). The anatomical location of the tumour had some dependence on the presence of this alteration as only one in nine tumours located in the temporal lobe bore a genetic defect, while half of frontal tumours presented $1 p / 19 q$ deletion (8/16, $P$ value for $\chi^{2}$ test equals 0.051 ).

Median time of follow-up was 65 months. Median time of patients' survival was 69.1 months (Fig. 2A). At the last follow-up, 10 patients were alive (29.4\%). Median time of survival differed among tumour types from 25 months in All, through 53.2 months in OAll, to 85.5 months in OII ( $p<0.04$, Fig. $2 \mathrm{~B})$.

Simultaneous deletion of $1 p / 19 q$ correlated with longer survival. Median survival for patients with and without this alteration was 80 and 41 months, respectively $(p<0.047)$ (Fig. 2C).

Among other demographic factors (sex, age at diagnosis) and clinical factors (affected hemisphere, frontal vs. other localization, adjuvant therapies), the only one having negative influence on the survival was frontal localization of the tumour $(p<0.046$, log-rank test) (Fig. 2D).

Therefore, two factors were chosen for the multivariate analysis - histological type and $1 \mathrm{p} / 19 \mathrm{q}$ deletion. Lack of $1 p / 19 q$ deletion correlated with an increased risk of death to a level of 2.855 ( $\mathrm{Cl}$ : 1.040-7.839). Other factors did not correlate with time of survival.

\section{Discussion}

Loss of $1 p$ and $19 q$ chromosomal arms is one of the most important molecular signatures in the diagnosis of central nervous system tumours. It is quite specific to oligodendroglial tumours and due to lack of any other phenotype and genetic marker typical of these neoplasms it becomes of interest for pathologists.

Table I. Detailed information regarding loss of genetic material on chromosomal arms $1 p$ and 19q with regard to coexistence of these defects

\begin{tabular}{|lcccc|}
\hline & 1p deletion & 19q deletion & 1p/19 codeletion & No defect \\
\hline Astrocytoma & 2 & 2 & 0 & 8 \\
\hline Oligoastrocytoma & 4 & 6 & 4 & 4 \\
\hline Oligodendroglioma & 8 & 8 & 8 & 4 \\
\hline
\end{tabular}


A

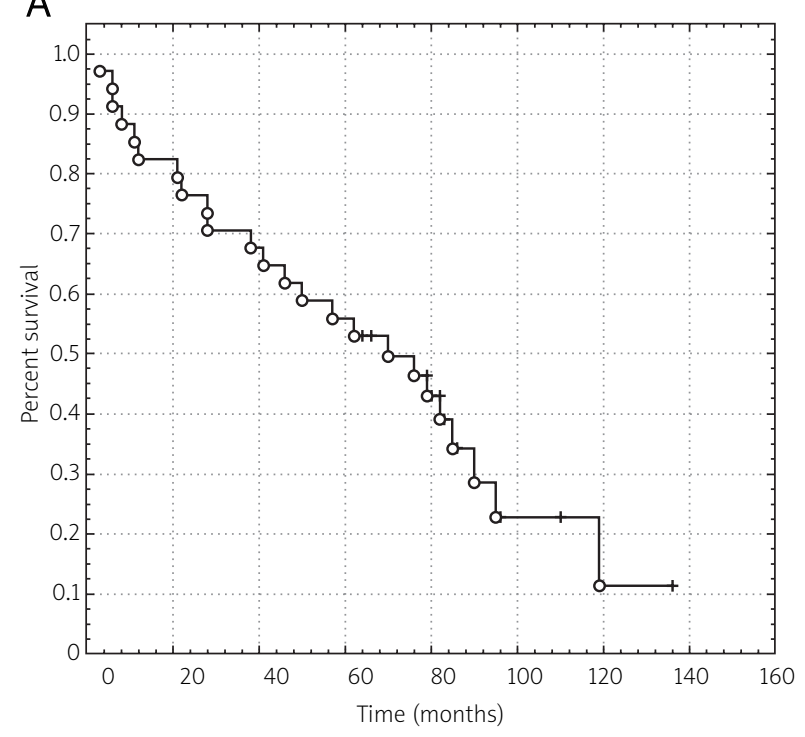

Observations:

- Complete

+ Censored

C

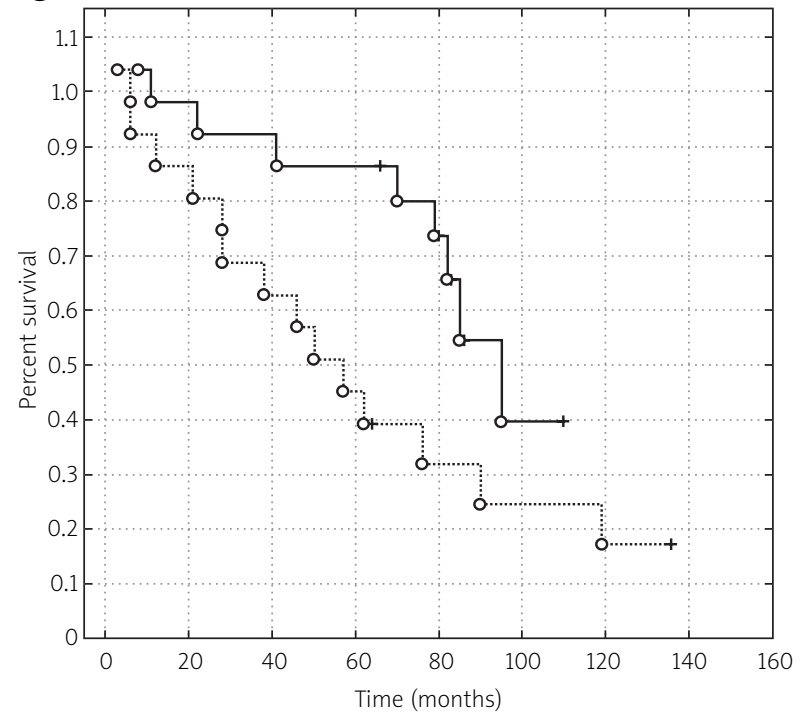

Observations: Location:

- Complete ....... Frontal lobe

+ Censored _ Any other location
B

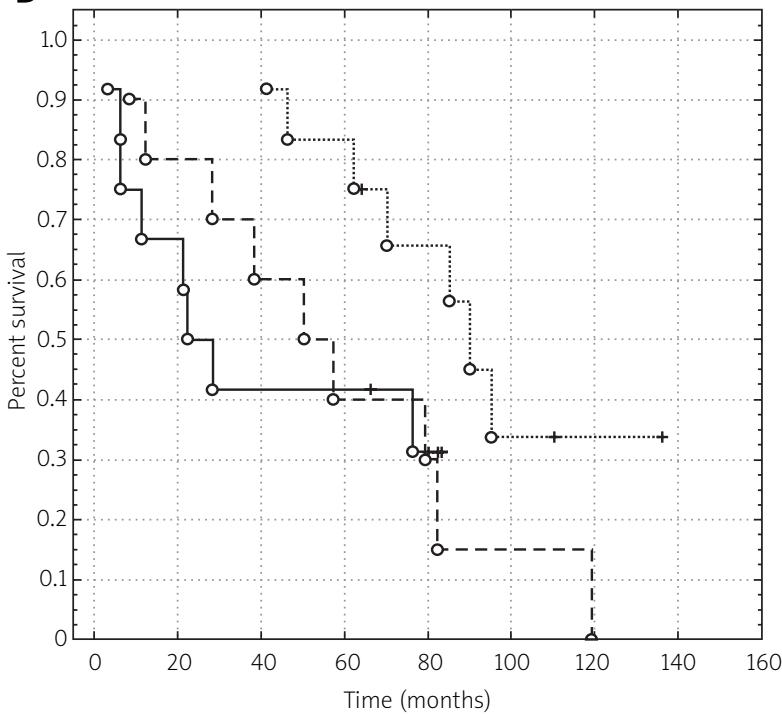

Observations: Type:

- Complete - A

+ Censored - - OA

D

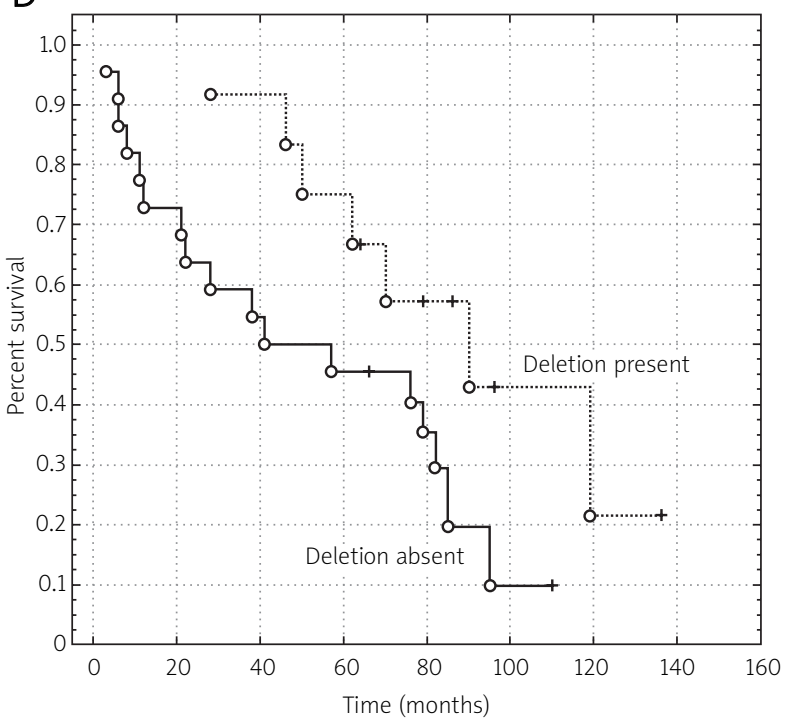

Observations: Deletion 1p/19q:

- Complete - N

+ Censored $\quad$........ Y

Fig. 2. Kaplan-Meier graphs depicting survivals of the whole examined group (A) and divided into subgroups. B) Survival influenced by histological type of tumour (A - astrocytoma, AO - oligoastrocytoma, O - oligodendroglioma). C) Comparison of two groups: one with tumour localized in the frontal lobe, second with different localization. The first group shows shorter survival, log-rank test, $p<0.05$. D) Group with loss of chromosomal arms $1 \mathrm{p} / 19$ as opposed to a group with unchanged chromosomes. The latter has shorter survival, confirmed by log-rank test, $p<0.05$. 
As the morphological features typical of neoplasms with oligodendroglial differentiation occur only in a subset of these tumours and they depend on the tissue processing, the diagnosis of these tumours is always a challenge. Loss of $1 p / 19 q$ seems to preferentially occur in tumours sharing these specific morphological features of oligodendroglial tumours (monomorphous cell population with 'fried egg' appearance, roundish nuclei). However, this classical histological presentation is present in a portion of oligodendroglial tumours. In others, the pathological diagnosis relies to a great extent on the subjective interpretation of a pathologist. Therefore, the diagnostic discrepancy is a true nightmare in the diagnosis of these neoplasms. Additionally, finding of the morphological features of oligodendrogliallike cells requires not only differentiation with tumours of astrocytic lineage but also other neoplasms having this phenotype (dysembryoplastic neuroepithelial tumour, neurocytoma) [1]. As determination of 1p/19q deletion presents also a prognostic and predictive value, analysis of this alteration is not of diagnostic importance but have therapeutic consequences. Therefore, we undertook this study to reveal its incidence in a group of diffuse LGGs.

The frequency of LGGs among all glial tumours we found was rather high as it amounted to $32.8 \%$. This value is in accordance with incidence [16]. Age at diagnosis is typical of this group of neoplasms and concordant with other published data [23].

There is no consensus with regard to the threshold of the oligodendroglial and astrocytic component required to diagnose mixed glioma. The WHO classification does not provide strict guidelines, either. This may constitute one of the causes of high differences in the number of oligoastrocytoma reported by other authors [22-24]. It can be explained by the fact that LGGs form a spectrum of pathological images, as presented by Ueki [32]. He proposed considering LGGs as a continuous spectrum of proliferative diseases occupied by astrocytoma and oligodendroglioma at two opposite ends and mixed glioma occupying its central part. Histological features do not make it possible to place a sharp border between described entities. An additional support to this thesis brings identification of IDH2 mutation shared by diffuse astrocytomas and oligodendrogliomas and regarded as an early molecular alteration in LGGs [21].

Analysis of both cellular components in mixed gliomas using microdissection, splitting astrocytic and oligo- dendroglial cell lines, provides evidence that both lines bear the same genetic defects. Seemingly, two subgroups may be identified among mixed gliomas [18]. One shows typical genotype for oligodendroglioma and the other - for astrocytoma $[18,26]$. Most importantly, both subgroups are indistinguishable on pathological examination. Therefore, more objective and precise markers are desirable to provide an optimal therapeutic approach to patients with these tumours.

As we showed and this observation supports earlier publications, deletions of chromosomal arms $1 p$ and $19 q$ are mutually strongly correlated $[23,24]$. We found simultaneous deletion of both chromosomal regions in $12 / 14$ cases ( $85.7 \%$ ) with $1 p$ deletion and $12 / 16$ cases $(75 \%)$ with $19 q$ deletion. Rarely, do these defects occur as independent alterations. Exclusive 19q deletion was found in diffuse astrocytomas and oligoastrocytomas and this is consistent with the results of previous surveys. Similarly, isolated deletion of $1 p$, if present in LGGs, was related to "pure" astrocytomas. We have not detected isolated deletions of either of these loci - all oligodendrogliomas presented with concurrent deletion of $1 p$ and 19q.

Since not all oligodendrogliomas demonstrate $1 p / 19 q$ deletion, morphological features that could help differentiate these two groups were deeply sought. In a study by Burger et al., 18 cases of LGGs were revised [5]. In each case when primary and revised diagnosis uniformly showed an oligodendroglial type, $1 p / 19 q$ deletion was found. On the other hand, when both diagnoses were inconsistent and astrocytoma was diagnosed during revision - this alteration was not identified. We presented very similar results. Therefore, we may conclude that when the tumour presents unequivocal morphological features of oligodendroglioma, $1 p / 19 q$ deletion may be suspected. Additional evidence is presented by Sasaki et al. [29]. In their paper published in 2002, oligodendrogliomas were divided into two groups - one including tumours of classic oligodendroglial image and the other having some astrocytoma features. Deletion of $1 p / 19 q$ was detected in $86 \%$ of tumours from the former group and $27 \%$ - in the latter group.

Among presumed predictive factors, three turned out to be statistically significant: histological type, $1 p / 19 q$ deletion and localization (frontal vs. other). These results are in concordance with literature data, which confirm strong influence of the first two factors on survival time [6,8,12,25]. 
We proved that simultaneous $1 p / 19 q$ deletion is a strong factor for better prognosis. If it occurs, the difference in median survival is 80 months in contrast to patients whose tumours do not present this alteration (41 months). This difference is independent of the histological type. These results justify genetic testing not only for oligodendrogliomas but for the whole group of LGGs.

Similarly to the other studies, we have noticed a correlation between $1 p / 19 q$ loss and location of the tumour. In addition to former reports that tumours with temporal, insular and diencephalic location usually correlated with the preservation of $1 p / 19 q$ chromosomal arms [33], we have found that tumours developing in the frontal lobe was of a much higher incidence of $1 \mathrm{p} / 19 \mathrm{q}$ loss.

Likewise, LGGs have age-dependent differences in occurrence of $1 p / 19 q$ deletion. Although histologically identical, WHO grade II diffuse gliomas developing in childhood usually do not show this alteration $[14,27]$. Due to the low incidence of LGGs in children, it is hard to evaluate influence of this genetic abnormality on survival. In our two youngest patients (case 40 aged 18 diagnosed with astrocytoma; case 60 aged 21 diagnosed with oligodendroglioma), this genetic defect was not found. Seemingly, childhood LGGs present other types of glial proliferation despite the similar histological presentation. Molecular alterations play an important role in this regard.

Other factors analysed in our group of patients did not correlate with survival time. It confirms the former publications, excluding age at diagnosis. Most of the authors noticed shorter survival in patients aged over 40. This observation probably did not result from differences in the treatment [25]. There are three possible explanations for this phenomenon. One is the clinical status of an elder patient who may suffer from comorbidities. Secondly, these patients usually present with more advanced neoplasms at the time of diagnosis, that directly results in increased tumour volume [2]. Moreover, aggressiveness of the gliomas developing in younger patients is seemingly lower. This is supported by a shorter time to progression [30], and lower proliferative capacity of the tumours in this age group of patients $[10,20]$.

Concluding, detection of $1 p / 19 q$ deletion in LGGs has two potential benefits. Firstly, due to its high incidence in oligodendrogliomas, it has a huge value as an additional tool in their correct and objective diag- nosis. As a consequence, this may be the first step for introduction of molecularly-based classification of glial tumours [17]. Secondly, this codeletion is a factor determining longer survival. Inclusion of a patient in a specific prognostic group may influence intensity of conducted treatment. The above features constituted the basis to include genetic examination in all patients with probable LGG in other countries. Results of the presented paper support application of $1 p / 19$ detection in common neurosurgical and pathological practice.

\section{Acknowledgments}

The paper was supported by the grant of the Ministry of Science No. ST-95 and W-791.

\section{References}

1. Aldape K, Burger PC, Perry A. Clinicopathologic aspects of $1 p / 19 q$ loss and the diagnosis of oligodendroglioma. Arch Pathol Lab Med 2007; 131: 242-251.

2. Bahary JP, Villemure JG, Choi S, Leblanc R, Olivier A, Bertrand G, Souhami L, Tampieri D, Hazel J. Low-grade pure and mixed cerebral astrocytomas treated in the CT scan era. J Neurooncol 1996; 27: 173-177.

3. Van den Bent MJ, Carpentier AF, Brandes AA, Sanson M, Taphoorn MJB, Bernsen HJJA, Frenay M, Tijssen CC, Grisold W, Sipos L, Haaxma-Reiche H, Kros JM, Van Kouwenhoven MCM, Vecht CJ, Allgeier A, Lacombe D, Gorlia T. Adjuvant Procarbazine, Lomustine, and Vincristine Improves Progression-Free Survival but Not Overall Survival in Newly Diagnosed Anaplastic Oligodendrogliomas and Oligoastrocytomas: A Randomized European Organisation for Research and Treatment of Cancer Phase III Trial. J Clin Oncol 2006; 24: 2715-2722.

4. Brada M, Viviers L, Abson C, Hines F, Britton J, Ashley S, Sardell S, Traish D, Gonsalves A, Wilkins P, Westbury C. Phase II study of primary temozolomide chemotherapy in patients with WHO grade II gliomas. Ann Oncol 2003; 14: 1715-1721.

5. Burger PC, Minn AY, Smith JS, Borell TJ, Jedlicka AE, Huntley BK, Goldthwaite PT, Jenkins RB, Feuerstein BG. Losses of chromosomal arms $1 p$ and $19 q$ in the diagnosis of oligodendroglioma. A study of paraffin-embedded sections. Mod Pathol 2001; 14: 842-853.

6. Cairncross G. Phase III Trial of Chemotherapy Plus Radiotherapy Compared With Radiotherapy Alone for Pure and Mixed Anaplastic Oligodendroglioma: Intergroup Radiation Therapy Oncology Group Trial 9402. J Clin Oncol 2006; 24: 2707-2714.

7. Cairncross JG, Ueki K, Zlatescu MC, Lisle DK, Finkelstein DM, Hammond RR, Silver JS, Stark PC, Macdonald DR, Ino Y, Ramsay DA, Louis DN. Specific genetic predictors of chemotherapeutic response and survival in patients with anaplastic oligodendrogliomas. J Natl Cancer Inst 1998; 90: 1473-1479.

8. Cavaliere R, Lopes MBS, Schiff D. Low-grade gliomas: an update on pathology and therapy. Lancet Neurol 2005; 4: 760-770.

9. Von Deimling A, Louis DN, Von Ammon K, Petersen I, Wiestler OD, Seizinger BR. Evidence for a Tumor Suppressor Gene on Chro- 
mosome 19q Associated with Human Astrocytomas, Oligodendrogliomas, and Mixed Gliomas. Cancer Res 1992; 52: 4277-4279.

10. Franzini A, Leocata F, Cajola L, Servello D, Allegranza A, Broggi G. Low-grade glial tumors in basal ganglia and thalamus: natural history and biological reappraisal. Neurosurgery 820; 35: 817-820.

11. Gadji M, Fortin D, Tsanaclis AM, Drouin R. Is the 1p/19q deletion a diagnostic marker of oligodendrogliomas? Cancer Genet Cytogenet 2009; 194: 12-22.

12. Kaloshi G, Benouaich-Amiel A, Diakite F, Taillibert S, Lejeune J, LaigleDonadey F, Renard MA, Iraqi W, Idbaih A, Paris S, Capelle L, Duffau H, Cornu P, Simon JM, Mokhtari K, Polivka M, Omuro A, Carpentier A, Sanson M, Delattre JY, Hoang-Xuan K. Temozolomide for low-grade gliomas: predictive impact of 1p/19q loss on response and outcome. Neurology 2007; 68: 1831-1836.

13. Kanamori M, Kumabe T, Sonoda Y, Nishino Y, Watanabe M, Tominaga T. Predictive factors for overall and progression-free survival, and dissemination in oligodendroglial tumors. J Neurooncol 2009; 93: 219-228.

14. Kreiger PA, Okada Y, Simon S, Rorke LB, Louis DN, Golden JA. Losses of chromosomes $1 p$ and $19 q$ are rare in pediatric oligodendrogliomas. Acta Neuropathol 2005; 109: 387-392.

15. Krouwer HGJ, Duinen SG, Kamphorst W, Valk P, Algra A. Oligoastrocytomas: A clinicopathological study of 52 cases. J Neurooncol 1997; 33: 223-238.

16. Louis D. and International Agency for Research on Cancer. WHO classification of tumours of the central nervous system. International Agency for Research on Cancer.

17. Louis DN, Holland EC, Cairncross JG. Glioma classification: a molecular reappraisal. Am J Pathol 2001; 159: 779-786.

18. Maintz D, Fiedler K, Koopmann J, Rollbrocker B, Nechev S, Lenartz D, Stangl AP, Louis DN, Schramm J, Wiestler OD, Von Deimling A. Molecular genetic evidence for subtypes of oligoastrocytomas. J Neuropathol Exp Neurol 1997; 56: 1098-1104.

19. Mariani L, Deiana G, Vassella E, Fathi AR, Murtin C, Arnold M, Vajtai I, Weis J, Siegenthaler P, Schobesberger M, Reinert MM. Loss of heterozygosity $1 \mathrm{p} 36$ and 19q13 is a prognostic factor for overall survival in patients with diffuse WHO grade 2 gliomas treated without chemotherapy. J Clin Oncol 2006; 24: 4758-4763.

20. McKeever PE, Junck L, Strawderman MS, Blaivas M, Tkaczyk A, Cates MA, Yan M, Li L. Proliferation index is related to patient age in glioblastoma. Neurology 2001; 56: 1216-1218.

21. Ohgaki H, Kleihues P. Genetic alterations and signaling pathways in the evolution of gliomas. Cancer Sci 2009; 100: 2235-2241.

22. Ohgaki H, Kleihues P. Population-based studies on incidence, survival rates, and genetic alterations in astrocytic and oligodendroglial gliomas. J Neuropathol Exp Neurol 2005; 64: 479-489.

23. Okamoto Y, Di Patre PL, Burkhard C, Horstmann S, Kleihues P, Jourde B, Fahey M, Schueler D, Probst-Hensch NM, Yasargil MG, Ohgaki H, Yonekawa Y. Population-based study on incidence, survival rates, and genetic alterations of low-grade diffuse astrocytomas and oligodendrogliomas. Acta Neuropathol 2004; 108 49-56.

24. Perry A, Fuller CE, Banerjee R, Brat DJ, Scheithauer BW. Ancillary FISH analysis for $1 p$ and 19q status: preliminary observations in 287 gliomas and oligodendroglioma mimics. Front Biosci 2003; 8: a1-9.
25. Pignatti F, Bent M van den, Curran D, Debruyne C, Sylvester R, Therasse P, Áfra D, Cornu P, Bolla M, Vecht C, Karim ABMF. Prognostic Factors for Survival in Adult Patients With Cerebral LowGrade Glioma. JCO 2002; 20: 2076-2084.

26. Qu M, Olofsson T, Sigurdardottir S, You C, Kalimo H, Nister M, Smits A, Ren ZP. Genetically distinct astrocytic and oligodendroglial components in oligoastrocytomas. Acta Neuropathol 2007; 113: 129-136.

27. Raghavan R, Balani J, Perry A, Margraf L, Vono MB, Cai DX, Wyatt RE, Rushing EJ, Bowers DC, Hynan LS, White CL. Pediatric oligodendrogliomas: a study of molecular alterations on $1 p$ and $19 q$ using fluorescence in situ hybridization. J Neuropathol Exp Neurol 2003; 62: 530-537.

28. Reifenberger J, Reifenberger G, Liu L, James CD, Wechsler W, Collins VP. Molecular genetic analysis of oligodendroglial tumors shows preferential allelic deletions on 19q and 1p. Am J Pathol 1994; 145: 1175-1190.

29. Sasaki H, Zlatescu MC, Betensky RA, Johnk LB, Cutone AN, Cairncross JG, Louis DN. Histopathological-molecular genetic correlations in referral pathologist-diagnosed low-grade "oligodendroglioma". J Neuropathol Exp Neurol 2002; 61: 58-63.

30. Shafqat S, Hedley-Whyte ET, Henson JW. Age-dependent rate of anaplastic transformation in low-grade astrocytoma. Neurology 1999; 52: 867-869.

31. Smith MT, Ludwig CL, Godfrey AD, Armbrustmacher VW. Grading of oligodendrogliomas. Cancer 1983; 52: 2107-2114.

32. Ueki K. Oligodendroglioma: Impact of molecular biology on its definition, diagnosis and management. Neuropathology 2005; 25: 247-253.

33. Zlatescu MC, TehraniYazdi A, Sasaki H, Megyesi JF, Betensky RA, Louis DN, Cairncross JG. Tumor location and growth pattern correlate with genetic signature in oligodendroglial neoplasms. Cancer Res 2001; 61: 6713-6715. 